\title{
A Class of High Resolution Difference Schemes Based on Non-Uniformly Cell Averaged-Solution Reconstruction
}

\author{
Chongkun $\mathrm{Xu}^{1, \mathrm{a}^{*}}$ and Yuting $\mathrm{X} \mathrm{u}^{2, \mathrm{~b}}$ \\ ${ }^{1}$ Nanchang Institute of Science and Technology, Nanchang, Jiangxi \\ ${ }^{2}$ Nanchang Institute of Science and Technology, Shangrao, Jiangxi, 330108, China \\ axuchongkunjx@126.com, b121037798@qq.com \\ * The Corresponding Author
}

\begin{abstract}
Keywords: Hyperbolic conservation laws; High-order accuracy; Difference scheme; Euler equations
\end{abstract}

Abstract. Based on non-uniformly cell averaged-solution reconstruction, a class of high-order accuracy and high resolution conservative difference schemes is obtained for one-dimensional nonlinear hyperbolic conservation laws in this paper. Its idea is the following. The First, the computational interval is divided into pieces of nonoverlapping sub-intervals, and then each sub-interval is further subdivided into small-intervals by using Gauss-Lobatto and Gauss-Chebyshev partitions according to required accuracy. Cell averaged-solutions from these small-intervals are used to reconstruct solutions at small-interval boundaries. Furthermore the correction is introduced. The second, the approximate Riemann solver is used to compute numerical fluxs at small-intervals boundaries, and a high-order accurate fully discretization method is obtained by applying high-order Runge-Kutta TVD time discretization. Moreover, the non-oscillatory property of the scheme is proved. The extension to systems is implemented. Finally, several typical numerical experients are given. The numerical results verify high accuracy and high resolution of the resulting schemes.

\section{Introduction}

As we all know, for the initial value problem of nonlinear hyperbolic conservation laws, even if the initial condition is a fully smooth function, its solution may also include discontinuities, such as shock wave and contact discontinuity. Therefore, studying its numerical method with high-order accuracy and high resolution to enable it to efficiently capture shock waves and these discontinuities and avoid generating numerical oscillations in the vicinity of shock and discontinuity has always been one of the key issues in the research of computational mathematics and computational fluid mechanics. In 1983, A.Harten proposed the TVD(Total Variation Diminishing)[1] difference scheme, which made a new breakthrough in the construction of high resolution and non oscillatory difference schemes. In recent years, some successful difference methods have been developed, such as ENO, WENO, DG and so on. In this paper, a class of high-precision conservative difference schemes based on the average reconstruction of non isometric cells for solving one-dimensional nonlinear hyperbolic conservation laws is given. Its structural thought is to divide the computation interval into non-overlapping small intervals and then the non isometric division is carried out for small intervals according to the accuracy requirement of the scheme. The polynomial approximation is carried out by the cell mean value in the small intervals in order to reconstruct the point value at the interface of small intervals and correct it. Then the approximate Riemann solution is used to calculate the numerical flux on the small interval interface to obtained the semi-discrete scheme of the spatial direction. And then the high-order Runge-Kutta TVD time discretization is carried out to obtain a class of full discrete difference scheme with high order accuracy. In addition, the non oscillatory characteristics of the scheme under certain CFL conditions are proved. Finally, the high resolution characteristics of the scheme are verified by several standard examples. 


\section{Construction of Difference Schemes}

One dimensional scalar nonlinear hyperbolic conservation law equation is considered.

$$
\left\{\begin{array}{l}
\frac{\partial u}{\partial t}+\frac{\partial f(u)}{\partial x}=0 \\
u(x, 0)=u_{0}(x)
\end{array}\right.
$$

Among them, $f^{\prime \prime}(u) \neq 0, t>0, u_{0}(x)$ is a periodic function or a function with tight support, $x \in[a, b]$.

Construct the difference scheme by the following steps:

The Division of Computational Intervals and the Definition of the Cell Mean Value. The computation interval $[a, b]$ is divided into $N$ small cells $I_{i}=\left[x_{i-1 / 2}, x_{i+1 / 2}\right](i=1,2, \cdots, N)$ that do not overlap each other. According to the requirement of the $k$-order accuracy of the scheme, each small cell $I_{i}$ is divided into $k$ non-isometric small cells $G_{i, j}=\left[x_{i, j-1 / 2}, x_{i, j+1 / 2}\right] \quad(j=1,2, \cdots, k)$, that is $I_{i}=\bigcup_{j=1}^{k} G_{i, j}$. Among them, the endpoint set $\left\{x_{i, j+1 / 2}\right\}$ of the small cell $G_{i, j}$ is Gauss-Lobatto point [8], point set $\left\{x_{i, j}\right\}$ is Gauss-Chebyshev point, that is:

$$
\begin{aligned}
& x_{i, j}=\left[x_{i+1 / 2}+x_{i-1 / 2}-\Delta x_{i, j} \cdot \cos \left(\left(j-\frac{1}{2}\right) \frac{\pi}{k}\right)\right] / 2 \quad(j=1,2, \cdots, k ; \quad i=1,2, \cdots, N), \\
& x_{i, j+1 / 2}=\left[x_{i+1 / 2}+x_{i-1 / 2}-\Delta x_{i, j} \cdot \cos \frac{j \pi}{k}\right] / 2 \quad(j=0,1,2, \cdots, k ; \quad i=1,2, \cdots, N),
\end{aligned}
$$

$\Delta x_{i, j}=x_{i, j+1 / 2}-x_{i, j-1 / 2}$, The subscript $i$ indicates the number of the small cells belonging to them.

The cell mean value of the $u(x, t)$ in the small cell $G_{i, j}$ is defined as:

$$
\bar{u}_{i, j}(t)=\frac{1}{\Delta x_{i, j}} \int_{x_{i, j-1 / 2}}^{x_{i, j+1 / 2}} u(x, t) d x
$$

The Reconstruction of the Point Value Based on the Cell Mean Value. In the small cell $I_{i}$, the cell mean value $\bar{u}_{i, j} \quad(j=1,2, \cdots, k)$ in $k$ small cells $G_{i, j}$ is used to construct the $k-1$-order interpolation polynomial $p_{i}(x)$ of $u(x, t)$, that is $p_{i}(x)=u(x)+O\left(h^{k}\right), x \in I_{i}$, so:

$$
\frac{1}{\Delta x_{i, j}} \int_{x_{i, j-1 / 2}}^{x_{i, j+1 / 2}} p_{i}(x) d x=\bar{u}_{i, j}
$$

Among them, $i=1,2, \cdots, N ; j=1,2, \cdots, k ; h=\sup _{\substack{1 \leq i \leq N \\ 1 \leq j \leq k}}\left\{\Delta x_{i, j}\right\}$.

The $k+1$ point values on $G_{i, j}$ boundary $x_{i, j+1 / 2}$ are reconstructed: $u_{i, j+1 / 2}=p_{i}\left(x_{i, j+1 / 2}\right)$ $=u\left(x_{i, j+1 / 2}\right)+O\left((\Delta x)^{k}\right)$. The values of the left and right sides of the $G_{i, j}$ boundary $x_{i, j+1 / 2}$ are respectively the $u_{i, j+1 / 2}^{\mp}$. For the boundary point of $G_{i, j}$ within $I_{i}, u_{i, j+1 / 2}^{ \pm}=p_{i}\left(x_{i, j+1 / 2}\right)$ is selected. For the left boundary of $I_{i}, u_{i, 1 / 2}^{-}=p_{i-1}\left(x_{i-1, k+1 / 2}\right)$ and $u_{i, 1 / 2}^{+}=p_{i}\left(x_{i, 1 / 2}\right)$ are selected. For the right boundary of $I_{i}, u_{i, k+1 / 2}^{-}=p_{i}\left(x_{i, k+1 / 2}\right)$ and $u_{i, k+1 / 2}^{+}=p_{i+1}\left(x_{i+1,1 / 2}\right)$ are selected. In order to avoid the numerical oscillation produced by the high order interpolation, the following correction is made:

$$
\tilde{u}_{i, j+1 / 2}^{-}=\bar{u}_{i, j}+m m\left(u_{i, j+1 / 2}^{-}-\bar{u}_{i, j}, \Delta \bar{u}_{i, j}, \Delta \bar{u}_{i, j-1}\right), \tilde{u}_{i, j+1 / 2}^{+}=\bar{u}_{i, j+1}-m m\left(\bar{u}_{i, j+1}-u_{i, j+1 / 2}^{+}, \Delta \bar{u}_{i, j+1}, \Delta \bar{u}_{i, j}\right)
$$

Among them, 


$$
m m(x, y, z)=\left\{\begin{array}{cc}
s \cdot \min (|x|,|y|,|z|), & x, y, z \text { 同号 } \\
0, & \text { 其它 }
\end{array}, s=\operatorname{sgn}(x), \Delta \bar{u}_{i, j}=\bar{u}_{i, j+1}-\bar{u}_{i, j} .\right.
$$

The Determination of the Numerical Flux on the Small Cell Boundary. The approximate Riemann resolver is used to calculate the numerical flux in $G_{i, j}$ boundary $x_{i, j+1 / 2}$. The following chooses LLF type flux[5]:

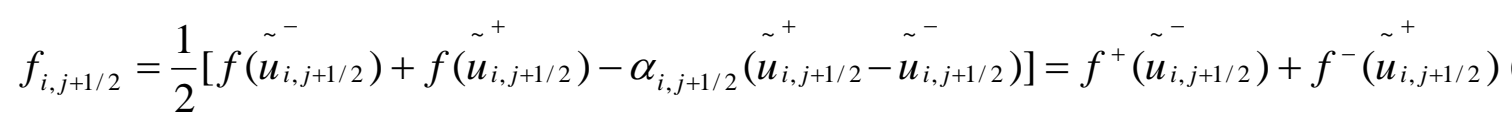

Among them, $f^{ \pm}(w)=\frac{1}{2}\left[f(w) \pm \alpha_{i, j+1 / 2} w\right], \quad \alpha_{i, j+1 / 2}=\underset{u}{\max }\left(\left|\frac{\partial f}{\partial u}\right|\right)$.

Fully Discrete Scheme. The semi discrete scheme of the spatial $k$-order accuracy of the approximation equation (1) on the small cell $G_{i, j}$ can be obtained from the above steps.

$$
\frac{\partial \bar{u}_{i, j}}{\partial t}=-\frac{f_{i, j+1 / 2}-f_{i, j-1 / 2}}{\Delta x_{i, j}} \stackrel{\Delta}{=} R(\bar{u})_{i, j}
$$

The fully discrete method(6) can be obtained by using the following third-order Runge-Kutta TVD time discrete method.

$$
\begin{aligned}
& \overline{-}_{i, j}^{(1)}=u_{i, j}^{n}+\Delta t \cdot R\left(u^{-n}\right)_{i, j}, \\
& \overline{-}_{i, j}^{(2)}=\frac{3}{4}{ }^{-n} u_{i, j}+\frac{1}{4} u_{i, j}^{(1)}+\frac{1}{4} \Delta t \cdot R\left(u^{-(1)}\right)_{i, j}, \\
& -_{i, j}^{n+1}=\frac{1}{3}{ }^{-n}{ }_{i, j}+\frac{2}{3}{ }^{-(2)}+\frac{2}{3} \Delta t \cdot R\left(^{-(2)}\right)_{i, j}
\end{aligned}
$$

The following results can be obtained:

Theorem 1. If CFL condition $\lambda \cdot \max _{u}\left|\frac{\partial f}{\partial u}\right| \leq \frac{1}{4}$ is satisfied, the fully discrete scheme (6) of the approximation equation (1) is the TVD scheme and satisfies the maximum principle in the average significance: $\max _{i, j}\left|\begin{array}{l}-n+1 \\ u_{i, j}\end{array}\right| \leq \max _{i, j}\left|\begin{array}{l}-^{n} \\ u_{i, j}\end{array}\right|$, among them, $\lambda=\frac{\Delta t}{\delta x}, \delta x=\min _{i, j}\left\{\Delta x_{i, j}\right\}$.

Prove: The first form of the scheme (6), that is the Euler forward time discrete scheme, is rewritten as the following incremental form:

$$
\bar{u}_{i, j}^{(1)}=\bar{u}_{i, j}^{n}+C_{i, j+1 / 2} \cdot\left(\bar{u}_{i, j+1}^{n}-\bar{u}_{i, j}^{n}\right)-D_{i, j-1 / 2} \cdot\left(\bar{u}_{i, j}^{n}-\bar{u}_{i, j-1}^{n}\right)
$$

Among them,

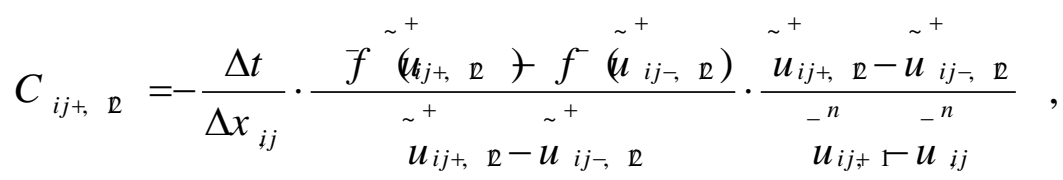

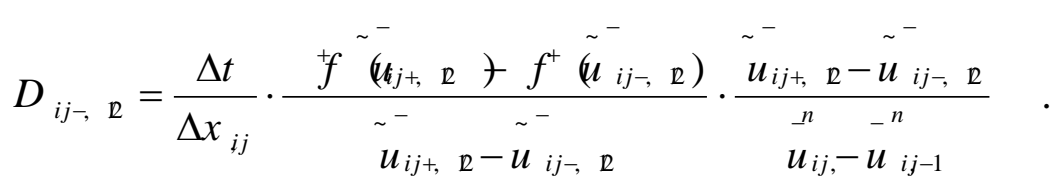

The formula(3) and formula (4) are put into the above formula, according to the definition of $m m(x, y, z)$ and $f^{ \pm}(u)$, we can obtain $C_{i, j+1 / 2} \geq 0, D_{i, j-1 / 2} \geq 0, \quad C_{i, j+1 / 2}+D_{i, j+1 / 2}$ 


$$
\leq 2 \lambda \cdot\left[\max _{u}\left(\frac{\partial f^{+}}{\partial u}\right)+\max _{u}\left(-\frac{\partial f^{-}}{\partial u}\right)\right] \leq 4 \lambda \max _{u}\left|\frac{\partial f}{\partial u}\right| \text {. }
$$

Therefore, when the CFL condition is satisfied, there is $C_{i, j+1 / 2}+D_{i, j+1 / 2} \leq 1$, so that we can get that the Euler forward time discrete scheme is the TVD scheme, and there is: $\max _{i, j}\left|\begin{array}{c}-{ }^{(1)} \\ u_{i, j}\end{array}\right| \leq \max _{i, j}\left|\overline{-}^{n}\right|$. The Runge-Kutta TVD time discrete scheme(6) is exactly the convex combination of Euler forward time discrete scheme, which proves that the fully discrete scheme (6) is TVD scheme, and satisfies the maximum principle: $\max _{i, j}\left|\bar{u}^{n+1}\right| \leq \max _{i, j}\left|\overline{-}^{n}\right|$.

\section{Equation Set Condition}

The initial value problem of the one-dimensional nonlinear hyperbolic conservation law equation is considered.

$$
\left\{\begin{array}{l}
\frac{\partial U}{\partial t}+\frac{\partial F(U)}{\partial x}=0 \\
U(x, 0)=U_{0}(x)
\end{array}\right.
$$

Among them, $F(U)=\left(f_{1}(U), f_{2}(U), \cdots, f_{m}(U)\right)^{T}, U=\left(u_{1}, u_{2}, \cdots, u_{m}\right)^{T}, \quad x \in[a, b]$.

Set the left and right eigenmatrix of the Jacobi matrix $A=\frac{\partial F(U)}{\partial U}$ of the flux function as $L$ and $R$. $L=\left(l^{(1)}, l^{(2)}, \cdots, l^{(m)}\right)^{T}$. Then we generalize the difference scheme obtained above to the one dimensional hyperbolic conservation law equation set. The specific steps are as follows:

It is the same as the step (1) of the scalar case.

In small cell $I_{i}$, the interpolation polynomial $p_{i}(x)$ is constructed in the same way as the scalar case (2). And also the value $u_{i, j+1 / 2}^{\mp}$ of the left and right sises of the boundary points of the $G_{i, j}$ inside the cell $I_{i}$ and the left and right boundary points of the $I_{i}$ is selected. The following correction is made for each characteristic variable $v=L u$ according to the scalar case:

$$
\begin{aligned}
& \left(v_{i, j+1 / 2}^{-}\right)^{(s)}=l_{i, j+1 / 2}^{(s)} \bar{u}_{i, j}+m m\left(l_{i, j+1 / 2}^{(s)} \cdot\left(u_{i, j+1 / 2}^{-}-\bar{u}_{i, j}\right), l_{i, j+1 / 2}^{(s)} \cdot \Delta \bar{u}_{i, j}, l_{i, j+1 / 2}^{(s)} \cdot \Delta \bar{u}_{i, j-1}\right) \\
& \left(v_{i, j+1 / 2}^{+}\right)^{(s)}=l_{i, j+1 / 2}^{(s)} \bar{u}_{i, j+1}-m m\left(l_{i, j+1 / 2}^{(s)} \cdot\left(\bar{u}_{i, j+1}-u_{i, j+1 / 2}^{+}\right), l_{i, j+1 / 2}^{(s)} \cdot \Delta \bar{u}_{i, j+1}, l_{i, j+1 / 2}^{(s)} \cdot \Delta \bar{u}_{i, j}\right) \\
& \text { Among them, } s=1,2, \cdots, m \text {. It is also recorded as } \\
& \tilde{u}_{i, j+1 / 2}^{ \pm}=R_{i, j+1 / 2}\left[\left(v_{i, j+1 / 2}^{ \pm}\right)^{(1)}, \cdots,\left(v_{i, j+1 / 2}^{ \pm}\right)^{(m)}\right]^{T} \text {. }
\end{aligned}
$$

In the s-th feature domain, the approximate Riemann solution operator of the scalar case is used for $l_{i, j+1 / 2}^{(s)} f\left(\tilde{u}_{i, j+1 / 2}^{ \pm}\right)$, then we can get:

$$
h_{i, j+1 / 2}^{(s)}=\frac{1}{2}\left[l_{i, j+1 / 2}^{(s)} f\left(\tilde{u}_{i, j+1 / 2}^{+}\right)+l_{i, j+1 / 2}^{(s)} f\left(\tilde{u}_{i, j+1 / 2}^{-}\right)-\alpha_{i, j+1 / 2}^{(s)}\left(\left(v_{i, j+1 / 2}^{+}\right)^{(s)}-\left(v_{i, j+1 / 2}^{-}\right)^{(s)}\right)\right]
$$

Among them, $\alpha_{i, j+1 / 2}^{(s)}=\max _{i, j} \rho\left(A_{i, j+1 / 2}\right)$.

The numerical flux at the boundary of the small cell $G_{i, j}$ is defined.

$$
h_{i, j+1 / 2}=R_{i, j+1 / 2}\left(h_{i, j+1 / 2}^{(1)}, \cdots, h_{i, j+1 / 2}^{(m)}\right)^{T}
$$

In small cell $G_{i, j}$, a semi discrete scheme is obtained by calculation. 


$$
\frac{\partial \bar{U}_{i, j}}{\partial t}=-\frac{h_{i, j+1 / 2}-h_{i, j-1 / 2}}{\Delta x_{i, j}}=R(\vec{U})_{i, j},
$$

Then, the third-order Runge-Kutta TVD time discrete method is used to get the fully discrete method.

\section{Numerical Experiment}

Here we give several standard numerical examples to verify the high efficiency of this high precision scheme.

In the following calculating examples, The following examples are calculated by third-order spatial discretization and third-order Runge-Kutta TVD time time discrete schemes.

Example 1. The initial value problem of one dimensional inviscid Burgers equation[10].

Nonlinear scalar Burgers equation $\frac{\partial u}{\partial t}+\frac{\partial\left(u^{2} / 2\right)}{\partial x}=0$ is considered. The initial condition is: $u(x, 0)=0.5+\sin (\pi x)$. The boundary meets the periodic boundary conditions. The computation interval is $[0,2]$, and 90 grids are selected. Fig.1 gives the results calculated by the non-isometric division of the cell when $t=1.5 / \pi$, and compares it with the exact solution. In the figure, the fine solid line is the exact solution, and the small circle point is the numerical solution. It is clear from the figure that the scheme in this paper can capture shock waves smoothly.

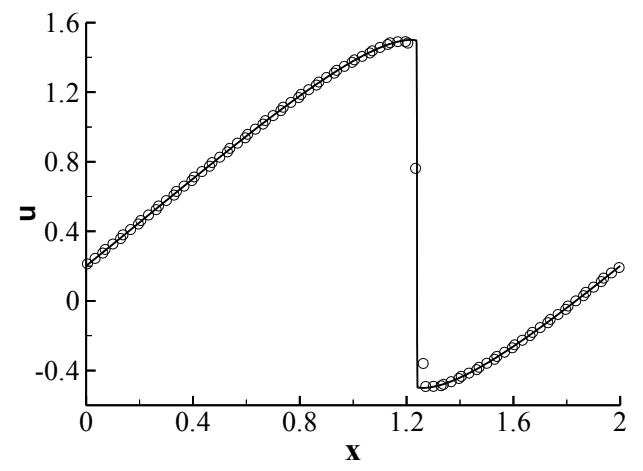

Figure 1. The calculation results of the Burgers equation when the time $t=1.5 / \pi$

Example 1. One dimensional shock tube problem

\section{Lax Problem}

The initial condition is: $(\rho, u, p)=\left\{\begin{array}{ll}(0.445,0.698,3.528), & x<0.5 \\ (0.5,0,0.571), & x \geq 0.5\end{array}\right.$.

The computation interval is taken as $[0,1]$, the characteristic decomposition method is used and 300 grids are selected. Fig. $2(a, b)$ is the result of the density and velocity of the Lax problem at the time of 0.15 respectively. In the figure, the fine solid line is the exact solution, and the small circle is the numerical solution. From the figure, we can see that the scheme in this paper has better ability to distinguish shock waves, contact discontinuities and rarefaction waves, and does not produce any oscillation near shock waves and contact discontinuities. 

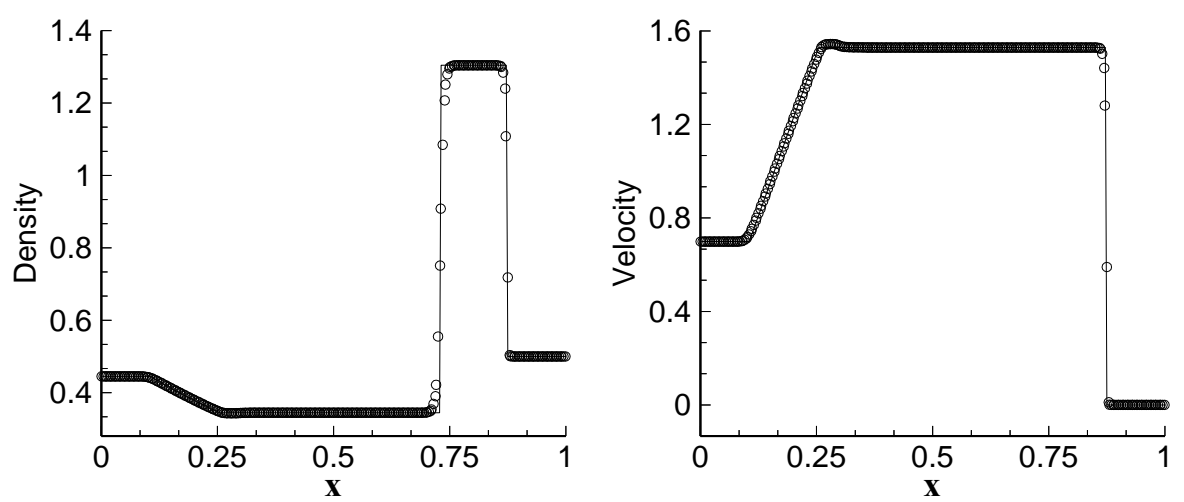

Figure2(a). Density distribution of Lax problem Figure 2(b). The velocity distribution of the Lax problem

\section{Conclusions}

The results of the above numerical calculation show that the high precision conservation difference scheme obtained in this paper has the characteristics of high efficiency resolution shock wave and contact discontinuity. In this paper, the calculation method of the subcell is used, and the same processing is made for each subcell, which can save more computational effort. In addition, the scheme in this paper is based on the reconstruction of the cell mean value, which is easily extended to the two-dimensional unstructured grid, and is used for the calculation of complex flow fields.

\section{Acknowledgements}

Teaching reform project of Nanchang Institute of Science\& Technology (NGJG-16-17)

\section{References}

[1] Harten A. High Resolution Schemes for Hyperbolic Conservation Laws [J], J. comput. Phys., 1983, 49: 357-393.

[2] Harten A, Engquist B, Osher S, Chakravarthy R. Uniformly High-Order Accurate Essential Non-Oscillatory Schemes III [J], J. Comput. Phys., 1987,71: 231-303.

[3] Shu C W ,Osher S. Efficient Implementation of Essential Nonoscillatory Shock Capturing Schemes [J], J. Comput .Phys., 1988,77:439-471.

[4] Shu C W ,Osher S. Efficient Implementation of Essential Nonoscillatory Shock Capturing Schemes II [J], J. Comput. Phys.,1989, 83:32-78.

[5] Liu X D ,Osher S. Convex ENO High Order Multidimensional Schemes without Field by Field Decomposition or Staggered Grids[J], J. Comput.Phys., 1998, 142:304-330.

[6] C.-W. Shu, Essentially Non-Oscillatory and Weighted Essentially Non-Oscillatory Schemes for Hyperbolic Conservation Laws, ICASE Report No.97-65.

[7] Cockburn B, Shu C W. TVB Runge-Kutta Local Projection Discontinuous Galerkin Finite Element Method for Conservation Laws II: General Framework[J]. Math. Comput., 1989, 52:411-435.

[8] D. Sidilkover, G.E. Karniadakis, Non-Oscillatory Spectral Element Chebyshev Method for Shock Wave Calculations, J. Comput. Phy., 1993, 107:10-22.

[9] W. Cai, D. Gottlieb, and A. Harten, Cell Averaging Chebyshev Methods for Hyperbolic Problems, in Comput. Math. Appl. Advances in PDE(Academic Press, London/New York, 1990).

[10] W.G.Zong, X.G.Deng and H.X.Zhang: Double Weighted Essentially Non-Oscillatory Shock-Capturing Schemes[J], Acta Aerodynamica Sinica, 2003, 21(2):218-225.(In Chinese) 